\title{
DISPROPORTIONATE AND INCREASING BURDEN OF HIV INFECTION AMONG MEN WHO HAVE SEX WITH MEN IN SlOVENIA: SURVEILLANCE DATA FOR 1999-2008
}

\author{
I Klavs (Irena.Klavs@ivz-rs.si) ${ }^{1}$, N Bergant ${ }^{1}$, Z Kastelic $^{1}$, A Lamut $^{1}$, T Kustec $^{1}$ \\ 1. AIDS, Sexually Transmitted Infections and Healthcare Associated Infections Unit, Communicable Diseases Centre, National \\ Institute of Public Health, Ljubljana, Slovenia
}

This article was published on 26 November 2009.

Citation style for this article: Klavs I, Bergant N, Kastelic Z, Lamut A, Kustec T. Disproportionate and increasing burden of HIV infection among men who have sex with men in Slovenia: surveillance data for 1999-2008. Euro Surveill. 2009;14(47):pij=19419. Available online: http://www.eurosurveillance.org/ViewArticle.aspx?ArticleId=19419

The report presents data on HIV infection among men who have sex with men (MSM) in Slovenia during 1999-2008. HIV surveillance was based on universal mandatory reporting of HIV/AIDS cases, monitoring HIV infection prevalence among sentinel populations of MSM and STI patients and selected behaviour indicators in a sentinel population of MSM. Among 48 newly diagnosed HIV cases reported for 2008, 34 were MSM. Since 1999, the annual reported rate of HIV diagnoses in MSM rose from 7.1 to 46.8 per million men aged 15-64 years (an increase of more than six times). During 1999-2008, the proportion of MSM diagnosed with AIDS within three months of HIV diagnosis declined from $60 \%$ to $21 \%$, however, the corresponding rate per million men aged 15-64 increased from 4.3 to 9.6. During 1999-2008, HIV prevalence among male clients of STI outpatient services tested for syphilis (including a substantial proportion of MSM) increased from $0 \%$ to $3.4 \%$, and it remained below $5 \%$ in a sentinel population of MSM in Ljubljana. In the same sentinel population of MSM, the proportion reporting HIV test last year increased from 29\% in 2003 to $38 \%$ in 2008 while the proportion reporting condom use at last anal intercourse decreased from $81 \%$ in 2004 to $66 \%$ in 2008 . The burden of HIV among MSM in Slovenia is disproportionately high and increasing fast. Promotion of safer sexual behaviour and HIV testing among MSM as well as positive prevention among MSM with diagnosed HIV infection are urgently needed.

\section{Introduction}

Human immunodeficiency virus (HIV) infection remains of major public health importance in Europe [1]. The predominant mode of transmission for HIV infection in European Union (EU) and European Free Trade Association (EFTA) countries appears to be sex between men [1]. The number of newly diagnosed HIV cases reported among men who have sex with men (MSM) has recently increased throughout EU and EFTA countries $[1,2]$. In 23 countries with consistent data for the period from 2000 to 2006, there was an overall $86 \%$ increase in the number of reported cases of newly diagnosed HIV infection among MSM [2]. Among these countries, in those reporting at least 20 new diagnoses of HIV infection among MSM in 2006, more than doubling of cases since 2000 was observed in five countries, with the highest increase of more than three times reported from Slovenia [2].

HIV surveillance data are vital to monitor the trends of the HIV epidemic and evaluate the public health response. Comprehensive
HIV surveillance including routine behavioural surveillance and HIV prevalence monitoring in addition to case-based national reporting systems for HIV and acquired immunodeficiency syndrome (AIDS) has been advocated for by the European Centre for Disease Prevention and Control (ECDC) and World Health Organization (WHO) Regional Office for Europe [1]. The main objective of the second generation HIV surveillance recommended by WHO and Joint United Nations Programme on HIV/AIDS (UNAIDS) is to monitor HIV and high-risk behaviour trends over time in order to provide essential data needed for the development of interventions and the evaluation of their impact $[3,4]$.

During 1999-2008, HIV surveillance in Slovenia, coordinated by the National Institute of Public Health (NIPH), has been based on routine collection, analysis and interpretation of data from:

- mandatory universal reporting of newly diagnosed cases of HIV infection, AIDS and deaths among AIDS cases;

- monitoring of overall HIV diagnostic testing rates;

- monitoring of HIV infection prevalence in several easily accessible sentinel populations at higher behavioural risk: MSM, patients with sexually transmitted infections (STI) and injecting drug users (IDU); and in one population group with on average low behavioural risk: pregnant women;

- monitoring HIV infection prevalence among blood donors by collating information on mandatory testing of all donated blood or blood products; and

- monitoring selected behaviour indicators in two easily accessible sentinel populations at higher behavioural risk: MSM and IDU $[5,6]$.

The aim of this paper is to present evidence of a disproportionate and increasing burden of HIV infection among MSM in Slovenia during the period from 1999 to 2008 using the second generation HIV surveillance data.

\section{Methods}

\section{Case reporting}

Reporting of HIV and AIDS has been mandatory in Slovenia since 1986. According to the Slovenian Communicable Diseases Act, physicians report anonymous individual data on newly diagnosed cases of HIV infection, AIDS and deaths among AIDS cases to the NIPH. European AIDS surveillance case definition has been used 
[7]. SOUNDEX coding of surnames together with dates of birth were used as unique identifiers to eliminate duplicates. Information collected included transmission category; the proportion of HIV cases categorised as other/unknown was $12 \%$ in 2008 and during the last ten years varied between the highest 39\% (in 1999) and the lowest 11\% (in 2005 and 2007). HIV diagnosis was defined as late at CD4 cells count $<350 \mathrm{~mm}^{3}$; very late at CD4 cells count $<200 \mathrm{~mm}^{3}$; and extremely late when AIDS clinical stage was reported within three months of HIV diagnosis.

\section{Monitoring diagnostic HIV testing}

To complement and better interpret information from universal mandatory reporting of newly diagnosed cases of HIV infection, the NIPH monitored overall HIV diagnostic testing rates by annual postal surveys including all Slovenian laboratories performing HIV testing. Only information on overall absolute numbers of HIV tests performed and numbers of positive test results was collated.

\section{Monitoring HIV infection prevalence in sentinel populations}

Detailed methods for monitoring HIV prevalence in selected sentinel populations with unlinked anonymous testing (including laboratory HIV testing strategy) together with the results for the period from 1993 to 2002 have been published previously [6]. Ideally, monitoring HIV prevalence in the chosen sentinel populations should provide information with respect to all three major modes of HIV transmission: unprotected sexual intercourse with infected individuals, exposure to infected blood and infected mother-to-child [3]. At the NIPH we had chosen three sentinel populations at higher behavioural risk: MSM, STI patients tested for syphilis, and IDU entering substitution treatment and/or taking part in needle and syringe exchange harm reduction programmes. In addition, we have monitored HIV prevalence in a low risk sentinel population of pregnant women screened for syphilis, a population group which is more similar to the general population with respect to the level of risk for HIV transmission. We had decided to use unlinked anonymous testing because individuals with high-risk behaviour might be more inclined than those with lower-risk behaviour to refuse or avoid HIV testing and information on results from voluntary confidential testing might be biased $[3,8]$. Also, the logistics of such an approach is simple and the costs are relatively low. Here we briefly present the data collection methods for two sentinel populations, MSM and STI patients, the latter group

\section{F I G U R E 1}

Sentinel sites for HIV prevalence monitoring in MSM and STI patients using unlinked anonymous testing, Slovenia, 1999-2008

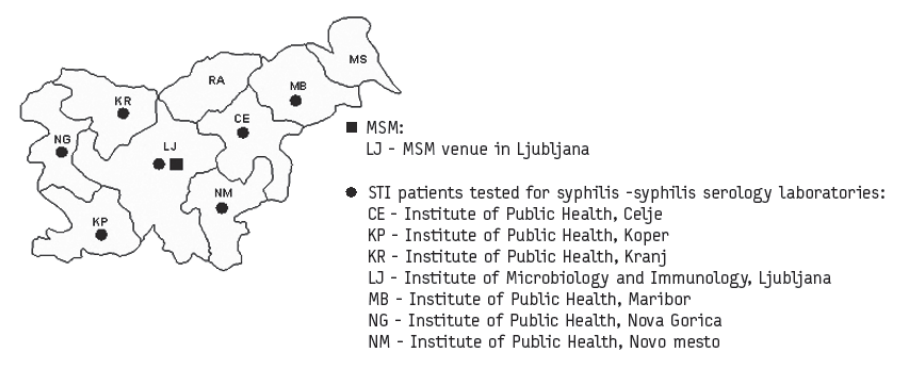

MSM: men who have sex with men

STI: sexually transmitted infections including a substantial proportion of MSM. Figure 1 presents the geographical distribution of corresponding sentinel sites.

Since 1993, residual sera from specimens obtained from patients of STI outpatient services and sent for syphilis serology have been sampled continuously and consecutively in all participating syphilis serology laboratories. The second inclusion of specimens obtained from the same individuals during the same calendar year was prevented by keeping a separate list of identifying information on individuals whose sera had already been included. All specimens were labelled only with information about the type of sentinel population, sampling period, sentinel site, gender and age group, frozen and stored at $-20^{\circ} \mathrm{C}$ until testing.

Since 1996, one-day cross sectional studies have been repeated annually by the NIPH in collaboration with a MSM non-governmental organisation (SKUC MAGNUS: 1996-2005; Legebitra: 20062008) at one sentinel site, a MSM community venue in the capital city Ljubljana. Saliva specimens have been voluntarily obtained from all men attending the event for unlinked anonymous testing for HIV surveillance purposes. All specimens were labelled only with information about the type of sentinel population, sampling period, sentinel site and age group. During these events, safer sex including condom use has been promoted and information about access to voluntary confidential or anonymous counselling and HIV testing has been provided.

\section{Monitoring selected behaviour indicators}

Since 2000, the NIPH has added a behavioural surveillance component to the HIV prevalence monitoring with unlinked anonymous testing in the sentinel population of MSM at a community venue in Ljubljana. All men participating and contributing a saliva specimen have also been invited to anonymously complete a short self-administered questionnaire. Behavioural data collected included information on condom use at anal sex, number of male partners and HIV testing during the year preceding the survey.

\section{Results}

Of a total of 48 newly diagnosed HIV infection cases reported for 2008 (23.5/million population), 34 cases, representing more than two thirds, were MSM. Since 1999, the annual reported rate of newly diagnosed HIV cases in MSM raised from 2.5 to 16.7 per million general population (Figure 2), which corresponds to 7.1 and 46.8 per million men 15-64 years old, an increase of more than six times. The overall increase in the number of newly diagnosed HIV cases in Slovenia during recent years has been due almost exclusively to the increase in new diagnoses among MSM.

During the same period, the proportion of MSM presenting with AIDS defining illness within three months of HIV diagnosis declined from $60 \%$ to $21 \%$ and the proportion of MSM with CD4 cells count $<200 / \mathrm{mm}^{3}$ at HIV diagnosis declined from $80 \%$ to $32 \%$ (Figure 3 ). However, the rate of extremely late diagnosis (AIDS within three months of HIV diagnosis) increased from 4.3 cases per million men 15 to 64 years old in 1999 to 9.6 cases per million men 15 to 64 years old in 2008 and the rate of very late diagnosis (presenting with CD4 cells count $<200 / \mathrm{mm}^{3}$ ) increased from 5.7 cases per million men 15 to 64 years old in 1999 to 15.1 cases per million men 15 to 64 years old in 2008.

In 2008, AIDS was diagnosed in seven MSM and in all seven cases clinical AIDS diagnosis was within three months of first HIV 
diagnosis. During the period from 1999 to 2008, the reported rates of AIDS diagnoses in MSM varied from 1.4 per million men 15 to 64 years old (in 2002) to 9.6 per million men 15 to 64 years old (in 2008). In 2008, death was reported for two MSM living with AIDS. During the period from 1999 to 2008, the corresponding death rates in MSM varied from 0 per million men 15 to 64 years old (in 2005 and 2006) to 2.9 per million men 15 to 64 years old (in 1999).

Overall HIV diagnostic testing rates (excluding unlinked anonymous testing for surveillance purposes and testing of all donations for blood and blood products safety purposes) remained relatively low during the last ten years, although an increase from
8.5 tests per 1,000 population in 1999 to 15.3 tests per 1,000 population in 2008 was observed. Overall, there were two positive test results per 1,000 HIV tests in 2008.

Some insight into recent changes in HIV testing uptake and sexual behaviour among MSM is provided by our sentinel behavioural surveillance in small convenience venue-based annual samples of MSM in Ljubljana. The proportion of MSM reporting an HIV test during the year preceding the survey increased from $29 \%$ in 2003 to $38 \%$ in 2008 , suggesting a slight improvement in HIV testing uptake in this particular sentinel population (Figure 4). Overall, our data on selected sexual behaviour indicators for the period from 1999 to 2008, do not suggest a major deterioration

F I G U R E 2

Newly diagnosed cases of HIV infection in Slovenia, 1999-2008: A) overall, among men, among MSM; B) according to transmission category

A

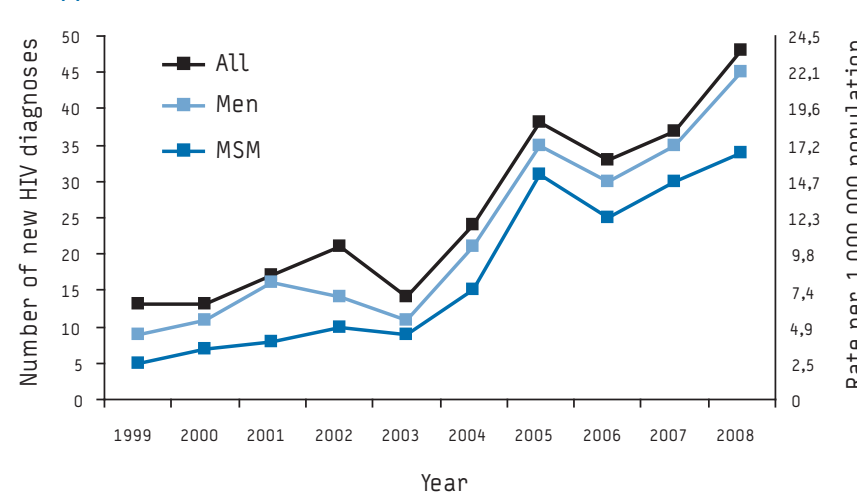

B

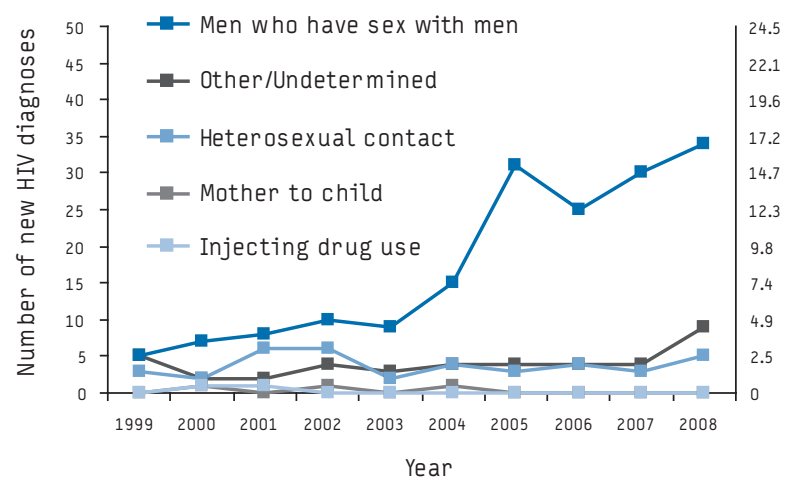

MSM: men who have sex with men

F I G U R E 3

Late diagnosis of HIV infection among MSM, Slovenia, 1999-2008

A

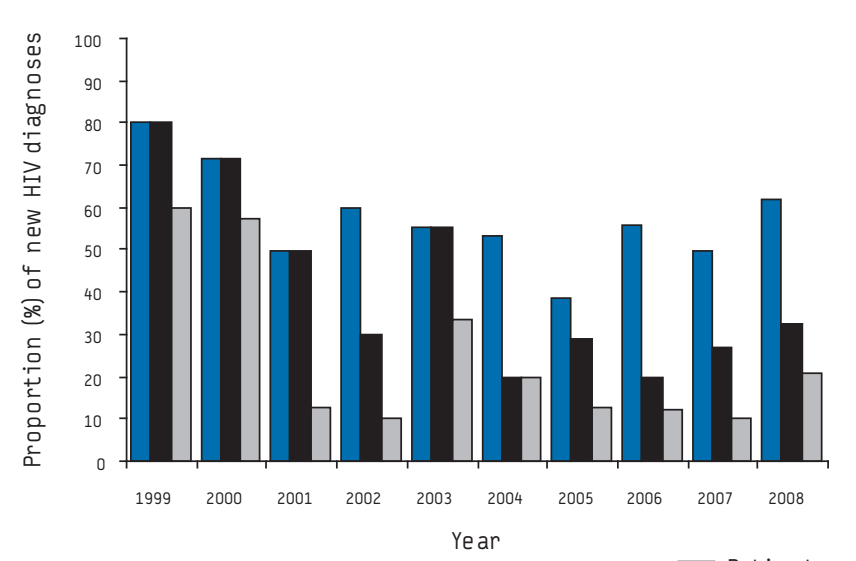

B

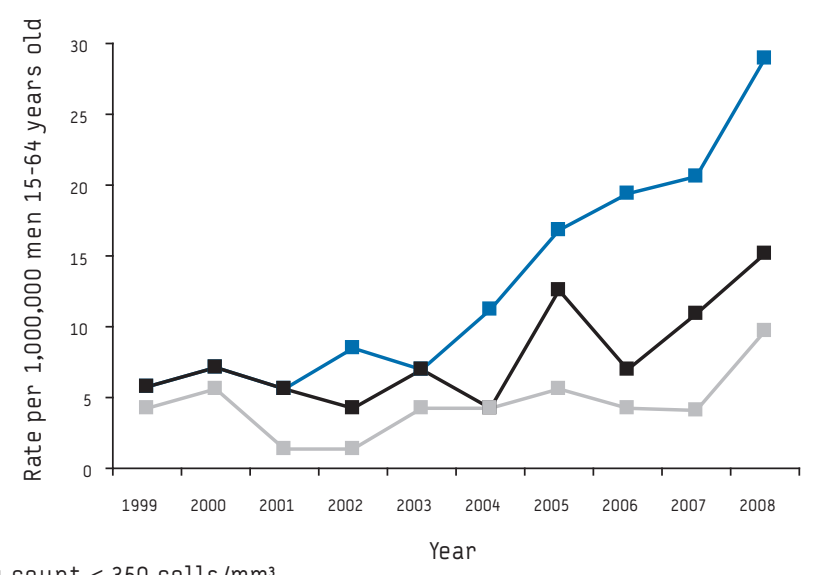

$\square$ Patients with CD4 count $<350$ cells $/ \mathrm{mm}^{3}$

- Patients with CD4 count < 200 cells $/ \mathrm{mm}^{3}$

$\square$ Patients with a clinical AIDS diagnosis within three months of HIV diagnosis 
of safe sexual behaviour in this particular sentinel population of MSM. The proportion of MSM reporting 10 or more male partners during the year preceding the survey has remained below $10 \%$ since 2005 and since 2004 at least half of MSM have reported to have always used a condom at anal intercourse during the year preceding the survey. However, in 2008, the proportion of MSM who reported to have never used a condom at anal intercourse during the year preceding the survey raised above $20 \%$ after being below $20 \%$ since 2004 and the proportion of MSM reporting to have used a condom during the most recent anal intercourse decreased from $81 \%$ in 2004 to $66 \%$ in 2008 , suggesting a recent slight deterioration in safe sexual behaviour among MSM in this particular sentinel population in Ljubljana.

During the period from 1999 to 2008, the prevalence of HIV in a national convenience sample of male clients of STI outpatient services tested for syphilis increased from 0\% in 1999 to $3.4 \%$ in 2008 (Table). A substantial proportion of male clients of STI outpatient services in Slovenia are MSM and early syphilis cases among MSM, including MSM with known HIV infection, represented a substantial proportion of all early syphilis diagnoses during recent years (data not shown) [9]. During the same period, the prevalence of HIV infection among female clients of STI outpatient services tested for syphilis remained below $1 \%$ and the prevalence of HIV infection consistently remained below $5 \%$ in a sentinel population of MSM in Ljubljana. For comparison, the prevalence of HIV infection among convenience samples of pregnant women

\section{F I G U R E 4}

Selected behavioural indicators in venue-based convenience samples of MSM, Ljubljana, Slovenia, 2001-2008

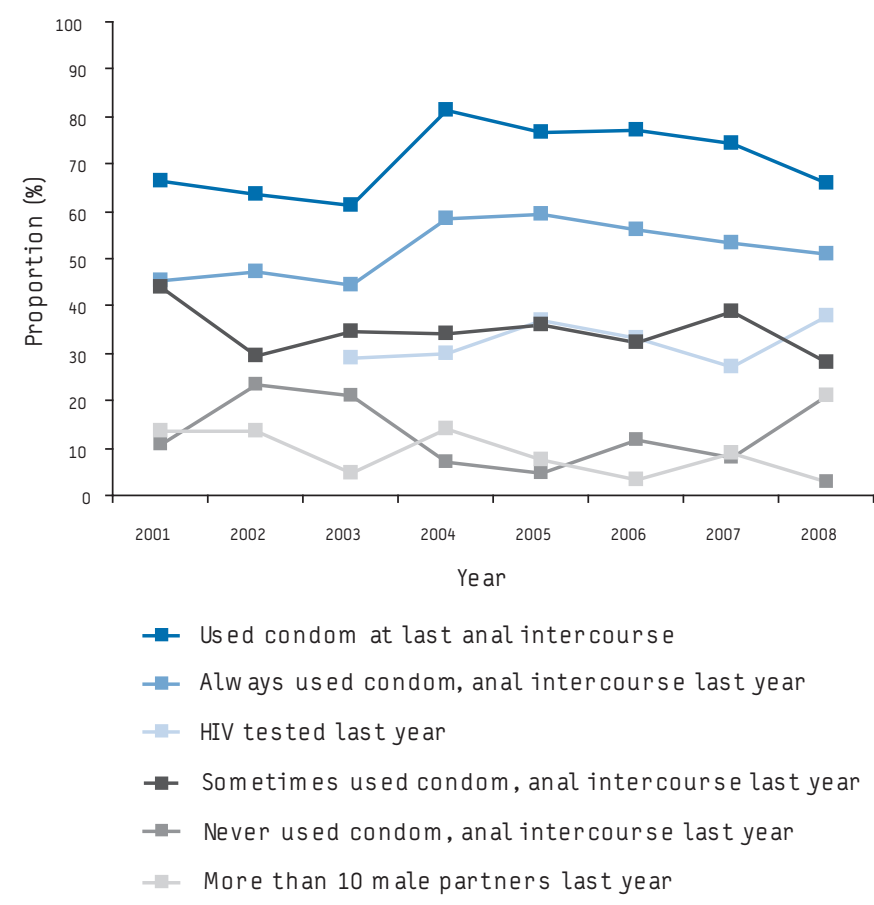

Note: The size of convenience samples of MSM recruited in 2001-2008 ranged from 68 in 2006 to 124 in 2008 (average: 89 ). The proportions shown in the Figure are based on the number of responses to individual questions (range: 59-124)

MSM: men who have sex with men screened for syphilis during the same period (sample sizes varied from the smallest 6,900 sera in 1999 to the largest 8,963 sera in 2007) varied from $0 \%$ (years: $2001,2003,2007$ ) to $0.01 \%$ (years: 1999, 2005).

\section{Discussion and conclusion}

The burden of HIV among MSM in Slovenia is disproportionately high and increasing fast. HIV cases among MSM represent the greatest proportion among all new HIV diagnoses and the substantial increase in the number of newly diagnosed HIV cases during recent years has been due almost exclusively to the increase in new diagnoses among MSM.

The former European Centre for the Epidemiological Monitoring of HIV/AIDS (EuroHIV) compared the annual burden of new HIV diagnoses among MSM calculated per 100,000 men 15 to 64 years old for European countries for the year 2006 [2]. For three countries these rates were above 100 per 100,000 , for 10 countries between 50 and 100 per 100,000 and for 14 below 50 per 100,000 . Slovenia with the rate of 35 newly diagnosed HIV cases in MSM per 100,000 men aged 15-64 in 2006, was in the third group. However, an analysis of trends in these reported rates for 23 countries with consistent HIV reporting systems showed an overall increase in 2006 in comparison to 2000 of $86 \%$ while for Slovenia the corresponding increase was $260 \%$.

Our national HIV surveillance data suggest that the recent increase in the reported numbers of newly diagnosed HIV cases among MSM in Slovenia may at least in part reflect a true increase in HIV incidence in this group and some deterioration of safer sex behaviour.

Some insight into recent changes in sexual behaviour among MSM is provided by our sentinel behavioural surveillance in small convenience venue-based annual samples of MSM in Ljubljana. Both the proportion of MSM who reported to have never used a condom at anal intercourse during the year preceding the survey and the proportion of MSM reporting to have not used a condom during the most recent anal intercourse increased in 2008, suggesting some deterioration of safe sexual behaviour among MSM in this particular sentinel population in Ljubljana. Recent increases in overall early syphilis reported rates in Slovenia (in 2008 increase of $130 \%$ compared with 2007), with great majority of cases occurring in men (94\%) and among cases in men a substantial proportion of cases among men known to have sex with men (44\%) and among those almost one in three with a foreign MSM partner within three months preceding syphilis diagnosis, suggest recent increase in unsafe sexual behaviour among MSM and sexual mixing of Slovenian MSM with MSM abroad [9]. Most worrying, two in three early syphilis cases reported in 2008 among MSM were men with known HIV infection, indicating unsafe sexual behaviour among HIV-positive MSM aware of their infection [9].

The overall HIV diagnostic testing rates in Slovenia have been relatively low in comparison to most EU countries [1], however it is not only important how many individuals are tested but also who is tested. The very simple method we use for monitoring national overall HIV testing rates does not provide data for understanding HIV testing rates in different patient groups (e.g. patients with tuberculosis or STI) or uptake of HIV testing in groups with different behavioural risk (e.g. MSM or IDU). So we do not have reliable information about whether HIV testing uptake has recently increased among MSM. However, our sentinel behavioural 
surveillance in small convenience venue-based annual samples of MSM in Ljubljana provides some insight into recent changes in HIV testing uptake and the data suggest a possible slight increase in HIV testing uptake in this particular sentinel population of MSM.

Relatively low reported AIDS incidence and mortality among MSM with AIDS in Slovenia can be attributed to universal access to treatment and care including highly active antiretroviral treatment (HAART) introduced after 1996. However, although our surveillance data for the last 10 years show a reduction in the proportion of extremely late HIV diagnoses (AIDS diagnosis within three months) among MSM, at least half of HIV infections diagnosed annually among MSM with the exception of year 2005, were late (CD4 cell count below $350 / \mathrm{mm}^{3}$ ), indicating that we have been missing opportunities for early treatment of HIV infection and positive prevention among MSM.

Our national HIV surveillance system with different components including behavioural surveillance and HIV prevalence monitoring in high-risk behaviour sentinel populations such as MSM and STI patients in addition to case-based national reporting systems for HIV and AIDS provides fairly good information about the distribution, trends in time and potential spread of HIV infection in Slovenia with rather modest investment of resources. It also provides public health decision makers with some insight into how successful HIV prevention, treatment and care interventions have been. The strengths of our monitoring of HIV infection prevalence in easily accessible sentinel populations of STI patients and MSM and monitoring selected behaviour indicators in a sentinel population of MSM in Ljubljana are minimal participation bias, anonymity, feasibility, sustainability and relatively low costs. The limitations include very small sample sizes to reliably monitor prevalence change and non-availability of additional risk behavioural information (e.g. history of sharing injecting equipment among MSM). We should be very cautious when inferring about the distribution and spread of HIV infection among all MSM and STI patients in Slovenia, as these easily accessible sentinel populations are not representative for all MSM and STI patients. Also the validity of self-reported behavioural data can always be questioned, but, if this bias does not change with time, such an approach is good for monitoring trends in self-reported behaviour among MSM. Overall, the results of our second generation HIV surveillance system are very informative and enable crude monitoring of trends in prevalence of HIV and selected behaviour indicators and early warning.

The burden of HIV among MSM in Slovenia is disproportionately high and increasing fast. Prevention and control of HIV and STI among MSM largely depends on supporting safe sexual behaviour among MSM, promoting uptake of HIV testing, and promoting HIV and STI healthcare seeking behaviour. Also, understanding sexual behaviour and needs of MSM with diagnosed HIV infection and positive prevention interventions among MSM are urgently needed.

\section{T A B L E}

HIV infection prevalence among MSM and STI patients, results of unlinked anonymous testing for surveillance purposes, Slovenia, 1999-2008

\begin{tabular}{|c|c|c|c|c|c|c|c|c|}
\hline & \multirow{2}{*}{ Year } & \multirow{2}{*}{ Number of sentinel sites } & \multicolumn{2}{|c|}{$\begin{array}{l}\text { Number of tested } \\
\text { individuals }\end{array}$} & \multicolumn{2}{|c|}{$\begin{array}{l}\text { Number of infected } \\
\text { individuals }\end{array}$} & \multicolumn{2}{|c|}{$\begin{array}{l}\text { Proportion of infected } \\
\text { individuals }\end{array}$} \\
\hline & & & Male & Female & Male & Female & Male & Female \\
\hline \multirow[t]{10}{*}{ MSM } & 1999 & 1 & 120 & & 2 & & $1.7 \%$ & \\
\hline & 2000 & 1 & 132 & & 4 & & $3.0 \%$ & \\
\hline & 2001 & 1 & 101 & & 3 & & $3.0 \%$ & \\
\hline & 2002 & 1 & 113 & & 0 & & $0 \%$ & \\
\hline & 2003 & 1 & 101 & & 1 & & $0.9 \%$ & \\
\hline & 2004 & 1 & 79 & & 2 & & $2.5 \%$ & \\
\hline & 2005 & 1 & 82 & & 3 & & $3.7 \%$ & \\
\hline & 2006 & 1 & 94 & & 2 & & $2.1 \%$ & \\
\hline & 2007 & 1 & 124 & & 3 & & $2.4 \%$ & \\
\hline & 2008 & 1 & 137 & & 3 & & $2.2 \%$ & \\
\hline \multirow[t]{10}{*}{ STI patients } & 1999 & 5 & 305 & 153 & 0 & 0 & $0 \%$ & $0 \%$ \\
\hline & 2000 & 6 & 279 & 107 & 0 & 0 & $0 \%$ & $0 \%$ \\
\hline & 2001 & 6 & 147 & 83 & 0 & 0 & $0 \%$ & $0 \%$ \\
\hline & 2002 & 7 & 334 & 201 & 1 & 1 & $0.3 \%$ & $0.5 \%$ \\
\hline & 2003 & 7 & 267 & 200 & 1 & 0 & $0.4 \%$ & $0 \%$ \\
\hline & 2004 & 7 & 328 & 148 & 5 & 0 & $1.5 \%$ & $0 \%$ \\
\hline & 2005 & 7 & 403 & 170 & 1 & 1 & $0.2 \%$ & $0.6 \%$ \\
\hline & 2006 & 7 & 419 & 211 & 10 & 0 & $2.4 \%$ & $0 \%$ \\
\hline & 2007 & 7 & 484 & 257 & 11 & 0 & $2.3 \%$ & $0 \%$ \\
\hline & 2008 & 7 & 677 & 264 & 23 & 2 & $3.4 \%$ & $0.8 \%$ \\
\hline
\end{tabular}


Finally, preventive responses targeted to MSM at the national level only may not be enough. MSM are highly mobile and there is substantial sexual mixing also between countries. Preventive interventions for MSM should probably be synchronised at the EU level and coverage, quality and impact of these programmes should be monitored on international level.

\section{Acknowledgements}

We thank all physicians for reporting diagnosed cases of HIV, AIDS and deaths among AIDS cases, especially from the Department of Infectious Diseases and Febrile Illnesses, University Medical Centre Ljubljana and from the Institute of Microbiology and Immunology (IMI), Medical School, University of Ljubljana. We also thank all healthcare organisations, regional institutes of public health, non-governmental organisations (NGOs) and IMI for participating in HIV prevalence monitoring and NGOs for participating in behavioural surveillance.

Financial support

Coordination of HIV and syphilis surveillance systems at the National Institute of Public Health is supported by the Slovenian Ministry of Health and Health Insurance Institute of Slovenia.

\section{References}

1. European Centre for Disease Prevention and Control, World Health Organization Regional Office for Europe. HIV/AIDS surveillance in Europe 2007. Stockholm: European Centre for Disease Prevention and Control; 2008. Available from: http://ecdc.europa.eu/en/publications/publications/0812_sur_hiv_aids_ surveillance_in_europe.pdf

2. Likatavicius G, Klavs I, Devaux I, Alix J and Nardone A. An increase in newly diagnosed HIV cases reported among men who have sex with men in Europe, 2000-6: implications for a European public health strategy. Sex Transm Inf. 2008;84(6);499-505.

3. World Health Organization (WHO) and Joint United Nations Programme on HIVIAIDS (UNAIDS). Guidelines for second generation surveillance. Geneva: WHO; 2000. Available from: http://www.emro.who.int/gfatm/guide/tools/ unaidssurveillance/unaidssurveillance.pdf

4. World Health Organization (WHO) and Joint United Nations Programme on HIV/AIDS (UNAIDS). Initiating second generation HIV surveillance systems: practical guidelines. Geneva: UNAIDS; 2002. Available from: http://www.who. int/hiv/pub/surveillance/en/isbn9291732192.pdf

5. Klavs I, Bergant N, Kastelic Z, Kustec T. [HIV infection in Slovenia]. Ljubljana: National Institute of Public Health; 2009. Slovene. Available from: http:// www.ivz.si/javne_datoteke/datoteke/1952-HIV_letno2008.pdf

6. Klavs I, Poljak M. Unlinked anonymous monitoring of human immunodeficiency virus prevalence in high- and low-risk groups in Slovenia, 1993-2002. Croat Med J. 2003;44(5):545-549.

7. European Centre for the Epidemiological Monitoring of AIDS. 1993 revision of the European AIDS surveillance case definition. AIDS surveillance in Europe. Quarterly Report No. 37. Paris: Institut de Médicine et d’Epidemiologie Africaines; 1993. Available from: http://www.eurohiv.org/reports/report_37/ aids_euro_definition_eng.pdf

8. Hull HF, Bettinger CJ, Gallaher MM, Keller NM, Wilson J, Mertz GJ. Comparison of HIV antibody prevalence in patients consenting to and declining HIVantibody testing in an STD clinic. JAMA. 1988;260(7):935-8.

9. Klavs I, Bergant N, Kastelic Z, Kustec T. [Sexually transmitted infections in Slovenia]. Ljubljana: National Institute of Public Health; 2009. Slovene. Available from: http://www.ivz.si/javne_datoteke/datoteke/930-SPO_letno2008. $\mathrm{pdf}$ 